\title{
Prediction of Some Thermodynamic Propertles of Selected Compounds of Element 104
}

\author{
Elijah Johnson* \\ Chemistry Division, Oak Ridge National Laboratory, P.O. Box 2008, Oak Ridge, Tennessee 37831-6375 \\ and $B$. Fricke \\ Department of Physics, University of Kassel, D-3500 Kassel, West Germany (Received: November 15, 1990)
}

\begin{abstract}
A set of parametrized equations has been published by Bratsch and Lagowski for calculating thermodynamic properties of the lanthanides, actinides, element 104, and certain related elements. Since these equations were applied to element 104, new values for the first four ionization energies and radii of the ions of charge $+1,+2,+3$, and +4 have been calculated for this element. The parametrized equations are used here with these new values to calculate some thermodynamic properties of element 104.
\end{abstract}

\section{Introduction}

In refs 1, 2, and 3 a method to calculate enthalpies and Gibbs free energies of formation is presented for some compounds of the actinide elements and element 104 and other transactinides. This method is based on a least-squares fit of data for the lanthanide elements and some related elements to an equation of prescribed form. The related elements are calcium, strontium, barium, yttrium, zirconium, hafnium, and thorium. The leastsquares parameters for the lanthanides were assumed to be suitable for use with the actinides and element 104. The calculational method is based on a knowledge of the heat of sublimation, ionization energies, and ionic radii of the metal element of interest. These required inputs arise from the use of the Born-Haber cycle to derive enthalpies and Gibbs free energies.

More accurate ionization energies and ionic radii are now available for element 104 than were available when the results in ref 3 were calculated. The main goal of this article is to present the changes in enthalpies and Gibbs free energies that result from using these more accurate parameters. The more accurate ionization energies and radii are based on multiconfiguration solutions of the Dirac-Fock equation of quantum mechanics. ${ }^{4}$

In section II the method used to calculate certain thermodynamic properties of element 104 is summarized. The ionization energies and ionic radii which are based on multiconfiguration solutions of the Dirac-Fock equation are presented. In section III results from using the new ionization energies and radii for deriving enthalpies and Gibbs free energies of formation are presented and compared with those in ref 3 .

\section{Method}

The equation used to calculate the enthalpy of formation of crystalline ionic compounds of the actinides and element 104 is presented in ref 3. Expressions for the entropy and Gibbs free energy are also presented there. Thermodynamic properties used for $\mathrm{H}^{+}$in the gas phase were obtained from ref 5 .

The standard electrode potential, $E^{\circ}$, for the following reaction

$$
\mathrm{M}^{n+}+[(n-m) / 2] \mathrm{H}_{2}=\mathrm{M}^{m+}+(n-m) \mathrm{H}^{+}
$$

for $n$ greater than $m$ is

$$
E^{\circ}=-\Delta G^{\circ} /[(n-m) F]
$$

where $\Delta G^{\circ}$ is the standard Gibbs free energy change for the reaction and $F=96.48456 \mathrm{~kJ} /(\mathrm{mol} \mathrm{V})$. The above reaction is denoted here by $\mathbf{M}^{n+} / \mathbf{M}^{m+}$. Here $M$ denotes a specific actinide element or element 104.

The value of the enthalpy of sublimation for element 104 that was used in ref 3 is $540 \mathrm{~kJ} / \mathrm{mol}$. The values for the first, second,

(1) Bratsch, S. G.; Lagowski, J. J. J. Phys. Chem. 1985, 89, 3310.

(2) Bratsch, S. G.; Lagowski, J. J. J. Phys. Chem. 1985, 89, 3317.

(3) Bratsch, S. G.; Lagowski, J. J. J. Phys. Chem. 1986, 90, 307.

(4) Desclaux, J. P. Comput. Phys. Commun. 1975, 9, 31.

(5) Bratsch, S. G.; Lagowski, J. J. J. Phys. Chem. 1984, 88, 1086.

To whom correspondence should be addressed. 
TABLE I: Calculated Ionic Radil in manometers of the $+1,+2,+3$, and +4 Ions of Element 104 by Extrapolation of the Radii of Maximum Charge Density of $(n-1)$ p Orbitals ${ }^{a}$

\begin{tabular}{cccccc}
\hline element & charge & RMAX & ionic radius 7,8 & $\alpha$ & $\beta$ \\
\hline $\mathrm{Ti}$ & +1 & 0.0481 & 0.096 & 0.0479 & \\
$\mathrm{Zr}$ & +1 & 0.0630 & 0.109 & 0.0460 & 0.0019 \\
$\mathrm{Hf}$ & +1 & 0.0631 & $(0.1082)$ & $(0.0451)$ & $(0.0009)$ \\
104 & +1 & 0.0172 & $(0.1164)$ & $(0.0452)$ & $(-0.0001)$ \\
$\mathrm{Ti}$ & +2 & 0.0481 & 0.086 & 0.0379 & \\
$\mathrm{Zr}$ & +2 & 0.0630 & $(0.0973)$ & $(0.0343)$ & $(0.0036)$ \\
$\mathrm{Hf}$ & +2 & 0.0632 & $(0.0959)$ & $(0.0327)$ & $(0.0016)$ \\
104 & +2 & 0.0710 & $(0.1041)$ & $(0.0331)$ & $(-0.0004)$ \\
$\mathrm{Ti}$ & +3 & 0.0476 & 0.0670 & 0.0194 & \\
$\mathrm{Zr}$ & +3 & 0.0626 & $(0.0784)$ & $(0.0158)$ & $(0.0036)$ \\
$\mathrm{Hf}$ & +3 & 0.0629 & $(0.0771)$ & $(0.0142)$ & $(0.0016)$ \\
104 & +3 & 0.0709 & $(0.0855)$ & $(0.0146)$ & $(-0.0004)$ \\
$\mathrm{Ti}$ & +4 & 0.0469 & 0.0605 & 0.0136 & \\
$\mathrm{Zr}$ & +4 & 0.0620 & 0.072 & 0.0100 & 0.0036 \\
$\mathrm{Hf}$ & +4 & 0.0626 & 0.071 & 0.0084 & 0.0016 \\
$\mathrm{l04}$ & +4 & 0.0706 & $(0.0794)$ & $(0.0088)$ & $(-0.0004)$
\end{tabular}

'The value of $n$ is equal to $4,5,6$, and 7 for titanium, zirconium, hafnium, and element 104, respectively. The values in parentheses are estimates.

third, and fourth ionization energies that were used there are 7.0, $13.0,24.0$, and $33.0 \mathrm{eV}$, respectively. These correspond to values for the sum of the first two, first three, and first four ionization energies of element 104 of $20.0,44.0$, and $77.0 \mathrm{eV}$, respectively. The value of the entropy of crystalline element 104 used in ref 3 was $48 \mathrm{~J} /(\mathrm{mol} \mathrm{K})$. In the calculations reported here the values of the enthalpy of sublimation and of the entropy of crystalline element 104 were the same as those used in ref 3. The sum of the first two, first three, and first four ionization energies of element 104 used here were $21.2,45.1$, and $76.8 \mathrm{eV}$, respectively. ${ }^{6}$ The calculated first ionization energy of element 104 is $6.5 \mathrm{eV} .^{6}$

The values used in ref 3 for the radii of the $+2,+3$, and +4 ions of element 104 are $0.100,0.087$, and $0.0820 \mathrm{~nm}$, respectively. The values for the ionic radii used here were obtained from the position of the maximum of the charge density, RMAX, in the $(n-1) p$ orbitals of titanium, zirconium, hafnium, and element 104 and an extrapolation procedure. The value of $\boldsymbol{n}$ for titanium, zirconium, hafnium, and element 104 is $4,5,6$, and 7, respectively. The quantity RMAX is the location of the maximum in $r^{2} R(r)^{2}$ where $R(r)$ is the radial part of the wave function for an orbital and $r$ is the distance from the origin. The values of RMAX for $\mathrm{Ti}, \mathrm{Zr}, \mathrm{Hf}$, and element 104 with charges of +1 to +4 are given in Table $I$.

The extrapolation procedure used to obtain radii of ions of element 104 and of some other ions is illustrated in Table I. In Table I an $\alpha$ value is equal to the difference between the corresponding values of the ionic radius ${ }^{7.8}$ and RMAX. For a given value of the charge the value of $\beta$ for an element is equal to the result of the value of $\alpha$ for this element subtracted from the value of $\alpha$ for the element above the specified element in Table I. For example, the value of $\beta$ for $\mathrm{Zr}^{+}$is equal to $(0.0479-0.0460) \mathrm{nm}$. $\beta$ values for element 104 were obtained by a linear extrapolation of these values for zirconium and hafnium. Experimental values of the ionic radii have been determined for all charge states of interest only for Ti. Experimental values of the radii for each of the three elements $\mathrm{Ti}, \mathrm{Zr}$, and $\mathrm{Hf}$ have been determined only for the +4 charge state. ${ }^{7}$ The extrapolation procedure gives values for the radii of the $+2,+3$, and +4 charge states of element 104 of $0.104,0.086$, and $0.079 \mathrm{~nm}$, respectively. The experimental values for ionic radii of +1 charge states were taken from ref 8 . Radii for other ions were taken from ref 7. Equation 1 of ref 3 gives predictions for a number of ionic radii of the first 14 members of the period 7 elements. Element 104 is the 17 th member of this

(6) Johnson, E.; Fricke, B.; Keller, O. L.; Nestor, C. W.; Tucker, T. C. J. Chem. Phys. 1990, 93, 8041 .

(7) Shannon, R. D. Acta Crystallogr. 1976, A32, 751.

(8) Dean, J. A., Ed. Lange's Handbook of Chemistry; McGraw-Hill Book Co.: New York, 1985; pp 3-121, 3-126.
TABLE II: Values of RMAX in nanometers of the $+1,+2,+3$, and +4 Ions of Group $4 \mathrm{~b}$ Elements for the $(n-2) f,(n-2) d,(n-1) \mathrm{s}$, $(n$ - 1)p, and $(n-1) d$ Orbitals ${ }^{a}$

\begin{tabular}{ccccccc}
\hline & & \multicolumn{5}{c}{ orbital } \\
\cline { 2 - 7 } element & charge & $(n-2) \mathrm{f}$ & $(n-2) \mathrm{d}$ & $(n-1) \mathrm{s}$ & $(n-1) \mathrm{p}$ & $(n-1) \mathrm{d}$ \\
\hline $\mathrm{Ti}$ & +1 & & & 0.0460 & 0.0481 & 0.0528 \\
$\mathrm{Zr}$ & +1 & & 0.0185 & 0.0572 & 0.0630 & 0.0851 \\
$\mathrm{Hf}$ & +1 & 0.0244 & 0.0252 & 0.0543 & 0.0631 & 0.1009 \\
104 & +1 & 0.0397 & 0.0341 & 0.0560 & 0.0712 & 0.1150 \\
$\mathrm{Ti}$ & +2 & & & 0.0461 & 0.0481 & 0.0523 \\
$\mathrm{Zr}$ & +2 & & 0.0185 & 0.0573 & 0.0630 & 0.0834 \\
$\mathrm{Hf}$ & +2 & 0.0244 & 0.0252 & 0.0545 & 0.0632 & 0.0872 \\
104 & +2 & 0.0397 & 0.0341 & 0.0560 & 0.0710 & 0.1158 \\
$\mathrm{Ti}$ & +3 & & & 0.0458 & 0.0476 & 0.0494 \\
$\mathrm{Zr}$ & +3 & & 0.0185 & 0.0571 & 0.0626 & 0.0798 \\
$\mathrm{Hf}$ & +3 & 0.0244 & 0.0252 & 0.0544 & 0.0629 & 0.0831 \\
104 & +3 & 0.0397 & 0.0341 & 0.0561 & 0.0709 & 0.0933 \\
$\mathrm{Ti}$ & +4 & & & 0.0453 & 0.0469 & \\
$\mathrm{Zr}$ & +4 & & 0.0185 & 0.0568 & 0.0620 & \\
$\mathrm{Hf}$ & +4 & 0.0244 & 0.0252 & 0.0543 & 0.0626 & \\
104 & +4 & 0.0397 & 0.0341 & 0.0561 & 0.0706 &
\end{tabular}

a The value of $n$ is equal to $4,5,6$, and 7 for titanium, zirconium, hafnium, and element 104 , respectively.

period. The values given by eq 1 of ref 3 imply that the ionic radius of the +1 charge state of element 104 should be close to the value given in Table $\mathbf{I}$.

The extrapolation procedure used here is based on ideas from finite-difference methods in numerical analysis. ${ }^{9}$ The independent variable is the principal quantum number, and the function of the principal quantum number of interest is the quantity $\alpha$. Three assumptions were used to obtain the radii of element 104 ions presented in Table I. The first assumption is that $\alpha$ is a quadratic function of the principal quantum number. This assumption implies that $[\beta(n)-\beta(n+1)]$ is the same for $n$ equal to 5 or 6 where $n$ is the value of the principal quantum number. The second assumption is that $[\beta(n)-\beta(n+1)]$ for the +1 charge state is proportional to $[\beta(n)-\beta(n+1)]$ for the +4 charge state. An assumption of this sort is necessary, because no experimental value for the radius of the +1 charged ion of hafnium is available. The third assumption is that, for each element, $\beta(n)$ for the +2 and +3 charge states are the same as $\beta(n)$ for the +4 charge state. This type of assumption is necessary, because no experimental values for the radii of the +2 and +3 charged ions of zirconium and hafnium are available. The first assumption is based on the periodic law of the chemical elements. Assumptions two and three are justified when uncertainties of suitable size are associated with the radii obtained by extrapolation. The uncertainties in the estimated ionic radii presented in Table $I$ are probably \pm 0.003 $\mathrm{nm}$. The uncertainty is assumed to be equal to one-half of the absolute value of the largest value of $\beta(n)$ plus one-half of the absolute value of $[\beta(n)-\beta(n+1)]$. The values in parentheses in Table I are estimates.

The $(n-1)$ p orbitals were used in the extrapolation procedure to find radii for element 104 , because these orbitals seem to yield more accurate values than $(n-2) \mathrm{f},(n-2) \mathrm{d},(n-1) \mathrm{s},(n-1) \mathrm{d}$, $n \mathrm{~s}$, or $n \mathrm{p}$ orbitals. There are two reasons for this. The first reason is that only the $(n-1) \mathrm{s}$ and $(n-1)$ p orbitals can yield third-order extrapolations. In principle, the $n s$ and $n$ p orbitals can also yield third-order extrapolations, but the required experimental radii for $\mathrm{Ti}, \mathrm{Zr}$, and $\mathrm{Hf}$ are not available to allow a third-order extrapolation for the radii of element 104 ions using these orbitals of the configuration functions used in the calculations, because values for RMAX for the $n s$ and $n p$ orbitals were not calculated for the +4 ions of $\mathrm{Ti}, \mathrm{Zr}, \mathrm{Hf}$, and element 104. As is indicated in Table II, these values of RMAX for the $(n-1) d$ orbitals were also not given by the calculations. The second reason is that the value of $\alpha$ is smaller with respect to RMAX for $(n-1)$ p orbitals than it is for $(n-1)$ s orbitals. This supports the assumption that

(9) Booth, A. D. Numerical Methods, 3rd ed.; Butterworths: London, 1966; pp 7-11. 
TABLE III: Calculated Standard Enthalpies of Formation of Some Element 104 (II) Compounds in $\mathrm{kJ} / \mathrm{mol}$ at $298.15 \mathrm{~K}$ and 1 -atm Pressure $^{a}$

\begin{tabular}{lrrr}
\hline compd & \multicolumn{1}{c}{ I } & \multicolumn{1}{c}{ II } & \multicolumn{1}{c}{ III } \\
\hline$(104) \mathrm{F}_{2}$ & -517 & -553 & -668 \\
$(104) \mathrm{Cl}_{2}$ & -96 & -122 & -238 \\
$(104) \mathrm{Br}_{2}$ & 16 & -9 & -125 \\
$(104) \mathrm{I}_{2}$ & 163 & 138 & 22 \\
$(104) \mathrm{O}$ & 80 & 37 & -79 \\
$(104) \mathrm{S}$ & 228 & 192 & 76
\end{tabular}

Column I: ionic radius $=0.104 \mathrm{~nm}$. Column II: ionic radius $=$ $0.100 \mathrm{~nm}$. Column III: results from ref 3 .

TABLE IV: Calculated Standard Enthalpies of Formation of Some Element 104 (III) Compounds in $\mathrm{kJ} / \mathrm{mol}$ at $298.15 \mathrm{~K}$ and $1-\mathrm{atm}$ Pressure $^{a}$

\begin{tabular}{crrr}
\hline compd & I & II & III \\
\hline$(104) \mathrm{F}_{3}$ & -1107 & -1086 & -1192 \\
$(104) \mathrm{Cl}_{3}$ & -399 & -377 & -484
\end{tabular}

${ }^{a}$ Column I: ionic radius $=0.086 \mathrm{~nm}$. Column II: ionic radius $=$ $0.087 \mathrm{~nm}$. Column III: results from ref 3 .

TABLE V: Calculated Standard Enthalpies of Formation of Some Element 104 (IV) Compounds in $\mathrm{kJ} / \mathrm{mol}$ at $298.15 \mathrm{~K}$ at 1 -atm Pressure $^{a}$

\begin{tabular}{crrr}
\hline compd & \multicolumn{1}{c}{ I } & \multicolumn{1}{c}{ II } & \multicolumn{1}{c}{ III } \\
\hline$(104) \mathrm{F}_{4}$ & -1896 & -1768 & -1748 \\
$(104) \mathrm{Cl}_{4}$ & -970 & -847 & -828
\end{tabular}

- Column I: ionic radius $=0.079 \mathrm{~nm}$. Column II: ionic radius $=$ $0.082 \mathrm{~nm}$. Column III: results from ref 3 .

radii obtained from $(n-1)$ p orbitals are more accurate than radii obtained from $(n-1) s$ orbitals.

In Table II the values of RMAX for the $(n-2) f,(n-2) \mathrm{d},(n$ $-1) \mathrm{s},(n-1) \mathrm{p}$, and $(n-1) \mathrm{d}$ orbitals are given. The values of the radii for element 104 obtained by extrapolation using $(n-$ 1)s orbitals for $104(1+), 104(2+), 104(3+)$, and $104(4+)$ are $0.1159,0.1004,0.0818$, and $0.0758 \mathrm{~nm}$, respectively. Even if these radii were used to obtain the thermodynamic results given here, the conclusions about the relative stabilities of the ions of element 104 would not change, because the value of the radius from the $(n-1)$ p orbital divided by the radius from the $(n-1)$ s orbital is greater for $104(4+)$ than for $104(3+)$ and it is greater for $104(3+)$ than for $104(2+)$.

\section{Results and Conclusions}

Standard enthalpies of formation for six compounds of element 104 are presented in Table III. The standard states are assumed to be crystalline with a specified structure. Results in column I of Table III were obtained by using the calculated electron ionization energies of section II of this report and the ionic radius presented in Table I for the +2 ion of element 104. Results in column II were obtained by using the calculated ionization energies of section $\mathrm{II}$ and the ionic radius for the +2 ion of element 104 which was presented in ref 3 . Results in column III were presented in ref 3 . The results in column I of Tables III-VII are probably more accurate than results in the other two columns of these tables. The effect of the changes in ionization energies may be seen by comparing results in columns II and III. For each compound considered in Table III the standard enthalpy of formation becomes less negative or more positive when the more accurate ionization energies are used. This means that these compounds are less stable than was implied by results in ref 3 .

Results for two +3 and +4 oxidation states compounds of element 104 are given in Tables IV and V. The effect of the change in ionic radius and ionization energies of element 104
TABLE VI: Calculated Standard Gibbs Free Energy of Formation of Element 104, +2, +3, and +4 Aqueous Ions, in $\mathrm{kJ} / \mathrm{mol}$ at $298.15 \mathrm{~K}^{a}$

\begin{tabular}{cccc}
\hline ion & I & II & III \\
\hline$(104)^{2+}$ & 144 & 109 & -2 \\
$(104)^{3+}$ & -83 & -62 & -169 \\
$(104)^{4+}$ & -453 & -331 & -312
\end{tabular}

${ }^{a}$ Column I: ionic radii are equal to $0.104,0.086$, and $0.079 \mathrm{~nm}$ for the $+2,+3$, and +4 ions, respectively. Column II: ionic radii are equal to $0.100,0.087$, and $0.082 \mathrm{~nm}$ for the $+2,+3$, and +4 ions, respectively. Column III: results from ref 3 .

TABLE VII: Calculated Standard Electrode Potentials in volts for Element 104 at $298.15 \mathrm{~K}^{a}$

\begin{tabular}{lrrr}
\hline electrode & I & II & III \\
\hline$(104)^{2+} /(104)$ & 0.7 & 0.6 & 0.0 \\
$(104)^{3+} /(104)$ & -0.3 & -0.2 & -0.6 \\
$(104)^{4+} /(104)$ & -1.2 & -0.9 & -0.8 \\
$(104)^{3+} /(104)^{2+}$ & -2.4 & -1.8 & -1.7 \\
$(104)^{4+} /(104)^{3+}$ & -3.8 & -2.8 & -1.5
\end{tabular}

${ }^{a}$ Column I: ionic radii are equal to $0.104,0.086$, and $0.079 \mathrm{~nm}$ for the $+2,+3$, and +4 ions, respectively. Column II: ionic radii are equal to $0.100,0.087$, and $0.082 \mathrm{~nm}$ for the $+2,+3$, and +4 ions, respectively. Column III: results from ref 3 .

causes +3 oxidation-state compounds to be less stable than is implied by results in ref 3 . These changes cause the +4 oxidation-state compounds to become relatively more stable. The values of ionization energies and radii used to obtain results in columns I, II, and III of Tables IV and V correspond to the values of these parameters used to obtain results in Table III. Calculated Gibbs free energies of formation of element $104,+2,+3$, and +4 aqueous ions are presented in Table VI.

Some calculated standard electrode potentials in volts for element 104 at $298.15 \mathrm{~K}$ are presented in Table VII. As was the case for the compounds, the new values for ionic radii and ionization energies used in this report cause the +2 and +3 oxidation states to become less stable with respect to the stability implied in ref 3 and they cause the +4 oxidation state to become relatively more stable.

The results obtained from new values for the ionic radii and ionization energies of element 104 support the general conclusions implied in ref 3 . These conclusions are that the +4 oxidation state of element 104 is significantly more stable than the +3 oxidation state which is more stable than the +2 oxidation state. The +1 oxidation state was not treated in ref 3 . The decreased relative stability of the +2 and +3 oxidation states of element 104 relative to the +4 oxidation state when compared with results in ref 3 is caused by the relatively larger two- and three-electron ionization potentials, relatively smaller four-electron ionization potential, and the relatively larger ionic radius for the +2 oxidation state, the slightly smaller radius for the +3 oxidation state, and the smaller radius for the +4 oxidation state. The uncertainties in the ionization potentials used in ref 3 varied from 1 to $2 \mathrm{eV}$. The results from the more accurate ionization potentials used here strengthen the conclusions presented above and in ref 3 .

Acknowledgment. We appreciate the advice and guidance provided by Dr. O. L. Keller, Jr., on the work presented. The research was sponsored by the Division of Chemical Sciences, Office of Basic Energy Sciences, U.S. Department of Energy, under Contract DE-ACO5-84OR21400 with Martin Marietta Energy Systems, Inc., the Deutsche Forschungsgemeinschaft (DFG), and the Gessellschaft fuer Schwerionenforschung (GSI), Darmstadt. The work was supported by the Florida State University Supercomputer Computations Research Institute which is partially funded by the U.S. Department of Energy through Contract DE-FC05-85ER25000. 